\title{
Simple and Approximately Optimal Pricing for Proportional Complementarities
}

\author{
YANG CAI, Yale University, USA \\ NIKHIL R. DEVANUR, Microsoft Research, USA \\ KIRA GOLDNER, University of Washington, USA \\ R. PRESTON MCAFEE, Microsoft, USA
}

\begin{abstract}
ACM Reference Format:
Yang Cai, Nikhil R. Devanur, Kira Goldner, and R. Preston McAfee. 2019. Simple and Approximately Optimal Pricing for Proportional Complementarities. In ACM EC '19: ACM Conference on Economics and Computation (EC '19), fune 24-28, 2019, Phoenix, AZ, USA. ACM, New York, NY, USA, 2 pages. https://doi.org/10.1145/ 3328526.3329562
\end{abstract}

We study a new model of complementary valuations, which we call "proportional complementarities." In contrast to common models, such as hypergraphic valuations, in our model, we do not assume that the extra value derived from owning a set of items is independent of the buyer's base valuations for the items. Instead, we model the complementarities as proportional to the buyer's base valuations, and these proportionalities are known market parameters.

Our goal is to design a simple pricing scheme that, for a single buyer with proportional complementarities, yields approximately optimal revenue. We define a new class of mechanisms where some number of items are given away for free, and the remaining items are sold separately at inflated prices. We find that the better of such a mechanism and selling the grand bundle earns a 12-approximation to the optimal revenue for pairwise proportional complementarities. This confirms the intuition that items should not be sold completely separately in the presence of complementarities. In the more general case, a buyer has a maximum of proportional positive hypergraphic valuations, where a hyperedge in a given hypergraph describes the boost to the buyer's value for item $i$ given by owning any set of items $T$ in addition. The maximum-out-degree of such a hypergraph is $d$, and $k$ is the positive rank of the hypergraph. For valuations given by these parameters, our simple pricing scheme is an $O(\min \{d, k\})$-approximation.

Proportional pairwise complementarities: We first define the PPC model. A single seller offers $m$ heterogeneous items for sale to a single buyer. We model the structure of the complementarities

A full version of this paper is available at https://homes.cs.washington.edu/ kgoldner/papers/proportionalcomplements.pdf.

Author's email addresses: yang.cai@yale.edu; nikdev@microsoft.com; kgoldner@cs.washington.edu; preston@mcafee.cc.

The work of Y. Cai was supported NSERC Discovery RGPIN-2015-06127 and FRQNT 2017-NC-198956. The work of K. Goldner was supported by a Microsoft Research PhD Fellowship as well as NSF grants CCF-1420381 and CCF-1813135.

Permission to make digital or hard copies of part or all of this work for personal or classroom use is granted without fee provided that copies are not made or distributed for profit or commercial advantage and that copies bear this notice and the full citation on the first page. Copyrights for third-party components of this work must be honored. For all other uses, contact the owner/author(s).

EC '19, June 24-28, 2019, Phoenix, AZ, USA

(C) 2019 Copyright held by the owner/author(s).

ACM ISBN 978-1-4503-6792-9/19/06.

https://doi.org/10.1145/3328526.3329562 
among the items via the following parameters, which are assumed to be known to the seller: ${ }^{1}$

$$
\eta_{i j} \in \mathbf{R}_{+} \quad \forall i, j \in[m], i \neq j .
$$

The parameter $\eta_{i j}$ captures how much having item $j$ boosts the valuation that the buyer derives from item $i$. The valuation of a buyer is determined by his type $t$, which is a vector in $\mathbf{R}_{+}^{m}$, and is the private information of the buyer. The $i^{\text {th }}$ coordinate of $t$ is $t_{i}$, which represents his base valuation for item $i$ in the absence of any other items. If the buyer also gets item $j$, then his valuation for item $i$ is boosted by an additional $\eta_{i j} t_{i}$. From this, we get that for any bundle $S \subseteq[m]$, the buyer's valuation for $S$ is

$$
v(t, S):=\sum_{i \in S} \eta_{i}(S) t_{i}, \quad \text { where } \quad \eta_{i}(S)=1+\sum_{j \in S \backslash\{i\}} \eta_{i j} .
$$

Note that $\eta_{i j}$ need not be equal to $\eta_{j i}$, and asymmetric boosts are only more general. We make the Bayesian assumption that $t$ is drawn from a product distribution $\Pi_{i \in m} F_{i}$. The distributions $F_{i}$ for all $i \in[m]$ (as well as the parameters $\eta_{i j}$ ) are known to the seller.

This more general asymmetric case corresponds to directed graphs (and hypergraphs). Thus we define the directed-positive-rank $k$ of the graph to be the maximum size of (number of items in) the source of a (hyper)edge. Thus, for the pairwise case, $k=1$.

The general case: The general class of valuations we consider is defined formally in the full paper; we give an informal description here. First of all, we allow hyperedges, instead of edges, i.e., each pair of item $i$ and a disjoint set of items $T$ forms a directed hyperedge $(T, i)$ and has a certain boost associated with it, denoted by $\eta_{i T}$ : this is the boost of having all items in $T$ on item $i$. The valuation of a set $S$ now includes all possible boosts due to hyperedges $(T, i)$ for $T \sqcup\{i\} \subseteq S$. We call this class of valuations proportional positive hypergraphic $(P P H)$ valuations. The other generalization is to allow the boost to be the maximum of the boost from multiple hypergraphs. We call this class of valuations maximum of proportional positive hypergraphic (МРPH) valuations. We denote by $k$ the directed-positive-rank and by $d$ the maximum-degree of the hypergraph.

Pricing scheme. Pricing the grand bundle is (conceptually) easy: set the monopoly price for the distribution of the buyer's value for the grand bundle, which can be computed from the given input. For simple valuations such as additive valuations, setting item prices to sell separately is also easy: set the monopoly reserve for each of them separately. In our model, this completely ignores the boost in the valuation on an item from having other items. Not surprisingly, this can be provably far from optimum when you have complementarities; we therefore need a non-trivial way to price the items in this case.

We introduce a class of mechanisms which we call SEPARATE/FREE. Like selling separately, every item is sold separately at some price, and the buyer may take any set of items so long as he pays the sum of their individual prices. However, we partition the items into "free items" $\mathcal{F}$, where for each item $i \in \mathcal{F}$, the individual price of each such item is $\$ 0$, and "priced items" $\overline{\mathcal{F}}=[m] \backslash \mathcal{F}$. Once the free set $\mathcal{F}$ is determined, we use the knowledge that the buyer will take the free items to inflate the monopoly prices of the priced items by the boost on the item from also getting the free set (and only the free set).

We show the following approximation guarantee more generally.

THEOREM 0.1 (INFORMAL). The better of BREV and the revenue from a mechanism of type SEPARATE/FREE is an $O(\min \{d, k\})$-factor approximation to the optimal revenue for valuations in the class МРPH. When $k=1$, i.e., the boosts are the maximum over directed graphs, the approximation factor is at most 12 .

\footnotetext{
${ }^{1}$ We use the notation $[m]$ to indicate the set of first $m$ natural numbers, $\{1,2, \ldots, m\}$.
} 